\title{
Concave rib osteotomy: a modified technique revisited
}

\author{
Mohamed A. El Masry · Ablaa M. Saleh · \\ Anthony B. McWilliams · E. Tsiridis · \\ Hossam Salah · Youssry K. El Hawary
}

Received: 15 July 2006/Revised: 3 April 2007 / Accepted: 20 May 2007/Published online: 15 June 2007

(C) Springer-Verlag 2007

\begin{abstract}
This is a prospective study reporting on the efficacy of a modified technique of Concave Rib Osteotomy (CRO) in conjunction with posterior instrumented fusion in the treatment of severe and rigid curves in patients with adolescent idiopathic scoliosis. The mean post-operative frontal plane correction was $68 \%$. The incidence of pulmonary complications was $11.5 \%$. The modified technique of CRO is a safe alternative to anterior release in treatment of severe and rigid curves.
\end{abstract}

Keywords Adolescent idiopathic scoliosis · Surgery · Concave rib osteotomy $\cdot$ Complications

\section{Introduction}

Scoliosis is a three-dimensional deformity of the spine which accounts for $80-85 \%$ of lateral spinal curves [9]. The prevalence data for patients with a Cobb angle of greater than 10 is between 0.5 and $3 \%$, the commonest being in the 12-14-year-old girls [11].

Severe and rigid curves usually require an anterior release and posterior instrumented fusion. The documented morbidity for a combined anterior and posterior approach is $10.2 \%$ and includes pulmonary, neurological and wound complications [3]. In addition the anterior approach has the

A. M. Saleh · H. Salah - Y. K. E. Hawary

Department of Orthopaedics,

Cairo University Teaching Hospitals, Cairo, Egypt

M. A. E. Masry $(\square) \cdot$ A. B. McWilliams .

E. Tsiridis

Department of Orthopaedics, Leeds Teaching Hospitals,

Leeds, West Yorkshire LS9 7TF, UK

e-mail: drmedoelmasry@yahoo.co.uk added potential for problems such as chylothorax, injury to the ureter, spleen, liver, or great vessels; and retroperitoneal fibrosis.

The concept of Concave Rib Osteotomy (CRO) was introduced by Flinchum in 1963 [4]. Kostuik, Goldstein, Tolo and Mann et al. have reported the use of such technique and the possible value as a release procedure $[5,7,8$, 12].

Halsall et al. [6] in cadaver studies tested flexibility before and after sectioning the ribs on the tension side, and found an average increase in deflection of $53 \%$.

However, the exact indications and the associated morbidity for this procedure have not been clearly identified in the literature. The aim of this study was to perform a prospective trial to assess the efficacy of using a modified $\mathrm{CRO}$ in conjunction with posterior instrumented fusion for the correction of adolescent idiopathic scoliosis.

\section{Materials and methods}

A prospective study of 78 patients undergoing a surgical correction for adolescent idiopathic scoliosis, all with severe and rigid curves measuring $70^{\circ}$ or more using the Cobb method [2] was conducted in a 5-year period between 1998 and 2003. Exclusion criteria: any etiology other than idiopathic, juvenile or infantile age groups, small and flexible curves.

There were 54 females and 24 males. The average age was 14.7 years (range $12-18$ years). The mean height was $140 \mathrm{~cm}$ (range $110-170 \mathrm{~cm}$ ). The mean weight was $46.4 \mathrm{~kg}$ (range $32-75 \mathrm{~kg}$ ).

Radiographic measurements were performed with the patient standing using coronal and lateral radiographs of the spine on $91 \mathrm{~cm}$ cassettes. Supine side bending films 
were used to assess flexibility of the curve. The flexibility index was calculated as a percentage of correction of the curve on the supine side bending from the original curve. All measurements were performed by one of the authors (AMS), who was independent of the operative team.

All patients had pulmonary function tests pre-operatively, 3 and 12 months post-operatively. All were performed on the same computerized spirometer (Chest graph HI-701 Chest M.I. Inc. Hongo, Bunkyo-Ku-Tokyo 113, Japan).

The tests were performed with the patient standing, each measurement was repeated three times, and the highest reading was selected.

All patients underwent a modified CRO in conjunction with a posterior instrumented fusion of the spine utilising iliac crest bone grafting. Hybrid Isola instrumentation with hooks, sublaminar wires, and screws (DePuy Acromed, Raynham, MA, USA) were used in all patients. A convex side costoplasty was performed in those with residual rib hump deformity following the final correction.

CRO involved an additional exposure which was carried lateral to the tips of the transverse processes of the vertebrae under consideration. We followed the description by Mann et al. [8] where a subperiosteal dissection proceeds in a plane directly over the ribs for $2-3 \mathrm{~cm}$. A rib cutter or Kerrison Rongeur was used to section the rib approximately $1 \mathrm{~cm}$ lateral to the transverse process. Our modifications include using a gauze swab underneath the osteotomised ribs to protect the underlying pleura, and creating a sling to lift the lateral ends of the ribs above the concave rod. We also filled the area with saline together with the use of positive ventilation to check for air leaks. No chest tubes were inserted routinely.

Post-operatively, all patients were mobilised without an orthosis. One week post-operatively, they received pulmonary rehabilitation program in the form of breathing exercises, incentive spirometer, treadmill and cycle ergometer for 3 days a week. This was followed 1 month later by abdominal weights resistant breathing exercises. The program was followed in all patients for 1-year postsurgery.

\section{Results}

The minimum follow-up was 24 months (range 24-30 months); none of the patients were lost to follow-up.

Pre-operatively the mean Vital Capacity [VC] was 3.21 (range $0.8-7.71$ ), and the mean forced expiratory volume in $1 \mathrm{sec}$ [FEV1] was 2.21 (range 0.2-5.8 1). One year postoperatively the mean [VC] was 3.41 (range 1.1-6 1), and the mean [FEV1] was 2.51 (range 0.6-5 1).
The mean pre-operative Cobb angle was $91.7^{\circ}$ (range $70^{\circ}-134^{\circ}$ ). The mean pre-operative flexibility index on supine side bending was $24 \%$ (range $17-29.5 \%$ ). The mean post-operative Cobb angle was $29.5^{\circ}$ (range $11^{\circ}-50^{\circ}$ ). This equates to a mean of $68 \%$ frontal plane correction.

Ten patients following the final correction and tightening of the rods underwent an additional convex side costoplasty for a residual rib hump deformity. A subperiosteal dissection was carried out over the convex side with osteotomy of the ribs; the resected parts of the ribs were used to augment the posterior fusion.

The overall incidence of pulmonary complications in all patients was $11.5 \%$ (9 patients).

The incidence of pulmonary complications in patients who underwent only CRO ( $n=68)$ was $10.2 \%$ (7 patients); two patients had air leaks from the pleural cavity intraoperatively. Post-operatively, three patients developed pneumothorax; and two patients had a pleural effusion.

The incidence of pulmonary complications in patients who underwent both CRO and convex side costoplasty ( $n=10)$ was $20 \%$ (2 patients), one patient developed pneumothorax; and one patient had a pleural effusion.

There were no post-operative wound complications and no neurological complications.

At 2-year follow-up, there was no evidence of pseudoarthrosis (Figs. 1a, b, 2a, b), but there was a mean loss of correction of $3^{\circ}\left(\right.$ range $\left.2^{\circ}-4^{\circ}\right)$.

\section{Discussion}

In the presence of a severe and rigid curve, a degree of spine flexibility is required in order to achieve a satisfactory curve correction. The options include a stand alone anterior release and instrumentation, combined anterior release with posterior instrumented fusion, or CRO with posterior instrumented fusion.

In a report of the scoliosis research society morbidity and mortality committee published by Coe et al. [3], they found that the overall complication rate for the 801 patients who underwent combined anterior release and posterior instrumented fusion to be $10.2 \%$ which corresponds to around double the complication rate of the anterior or posterior instrumentation alone. The incidence of pulmonary complications was $3.5 \%$.

The possible value of CRO as a release procedure has been previously reported in the literature both in studies on cadavers [6] and in clinical situations [5, 7, 8, 12].

However, there has been contradicting results regarding the associated morbidities [5, 8]. The addition of concave rib osteotomies to instrumentation and fusion procedures increases the risk of pulmonary morbidity. Goldstein [5] reported five pleural effusions and three pneumothoraces in 
Fig. 1 AP and lateral radiographs of an adolescent idiopathic scoliosis with a nine thoracic and lumbar curves
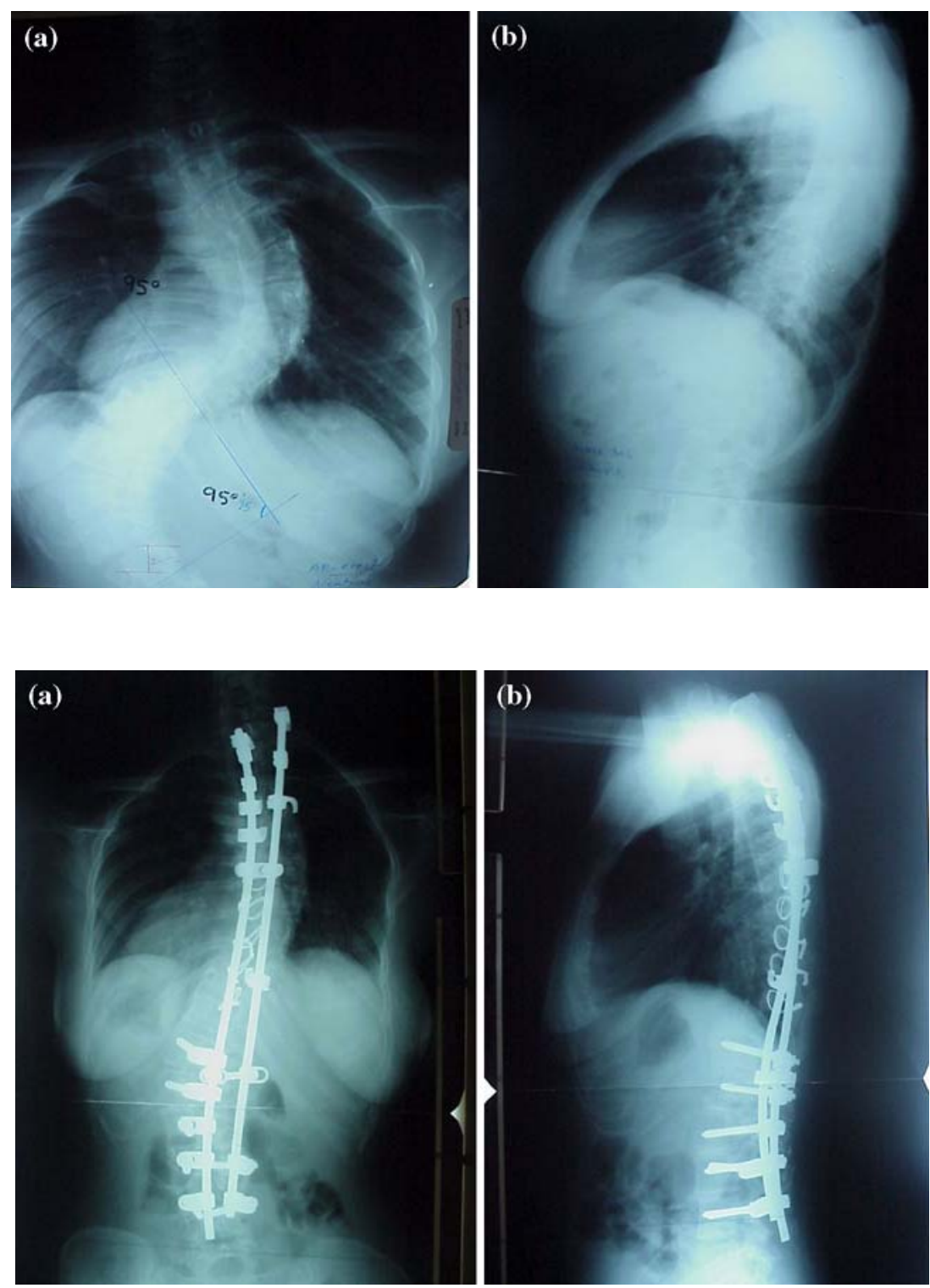

Fig. $2 \mathrm{AP}$ and lateral radiographs 2 years following posterior instrumented fusion with additional modified concave rib osteotomy
17 patients who had resection of $5-6 \mathrm{~cm}$ of concave ribs. Mann et al. [8] reported a decrease in the incidence of complications by performing rib osteotomies rather than rib resections. They reported two pleural effusions and one pneumothorax in 10 patients.

In our study, two patients had pleural tears intra-operatively that required chest drain insertion. Seven patients had post-operative pulmonary complications. Four out of seven had a post-operative pneumothorax; two of them required insertion of a chest drain. Three had pleural effusion that required drainage.

The overall pulmonary complications reported in this study were $11.5 \%$, which is higher than the pulmonary complication rate for anterior release. However this seemingly higher incidence of pulmonary complications is related to this particular group of patients with severe and rigid curves which required $\mathrm{CRO}$ and additional convex thoracoplasty in ten patients.

All previous reports on CRO have been on a small number of patients with different techniques utilised and on different types of curves resulting in a higher incidence of pulmonary complications $[5,8]$. To the best of our knowledge this is the first prospective study including 78 patients all diagnosed with adolescent idiopathic scoliosis reporting a lower incidence of pulmonary complications.

We believe that meticulous subperiosteal dissection with the help of a gauze swab, together with avoiding resection of the rib is mandatory. In addition, the use of saline placed over the pleura with positive ventilation intra operatively is a very useful tool to identify any possible leaks; which will require an insertion of a chest drain; thus reducing any associated pulmonary complications. 
The degree of frontal plane correction achieved in this study was $68 \%$, which matches most recent reports on combined anterior and posterior procedure, published in the literature [1].

The changes accompanying idiopathic scoliosis are summarized as; the apex of the curve directed to the right, the transverse processes are directed posteriorly and as a result, the ribs on the right side are widely separated, and cannot be moved further apart during inspiration, while on the left side, the ribs are crowded together. With further progression of the curve, this will result in rib hump on the convex side of the curve and depression of the ribs on the concave side [10].

The description of CRO by Mann et al. [8] was to lift the lateral rib segment with a Kocher clamp and allow it to posteriorly overlap the medial segment, our modified technique is to lift the lateral end of rib high above the rod which will contribute to the correction of thoracic cage asymmetry, and hence decreasing the need for an additional costoplasty on the convex side. In addition lifting the ribs will result in an increase in the antero-posterior chest dimensions.

The effect of CRO with posterior instrumentation on post-operative pulmonary functions has not been previously reported in the literature. In our study, despite the extended surgical dissection when using the modified CRO the chest wall dynamics appeared not to be altered with no statistically significant difference in the mean VC and FEV1 measured pre-operatively and one year post-operatively.

Weiner et al. [13] in a study following post-lung resection documented the effect of Pulmonary Rehabilitation Program (PRP) with achieving a significant increase in lung functions after 6 months post-operatively.

Finally CRO is utilised through the same posterior incision, thus avoiding the additional morbidity associated with anterior surgery.

\section{Conclusions}

We believe that intense PRP may have contributed to the return of normal pulmonary functions 1 year after surgery, but further studies are recommended to reach such a conclusion. The addition of the modified technique of CRO to posterior instrumented fusion in the treatment of severe and rigid curves in patients with adolescent idiopathic scoliosis is a safe and reliable alternative method to anterior release.

It achieved good correction and resulted in accepted post-operative complications.

However, the use of meticulous surgical techniques and adequate post-operative monitoring are mandatory in order to achieve such results.

\section{References}

1. Bullmann V, Halm HF, Schulte T, Lerner T, Weber TP, Liljenqvist UR (2006) Combined anterior and posterior instrumentation in severe and rigid idiopathic scoliosis. Eur Spine J 15:440

2. Cobb JR (1948). Outline for the study of scoliosis. Instr Course Lect 5:261-75

3. Coe JD, Arlet V, Donaldson W, Berven S, Hanson DS, Mudiyam R, Perra JH, Shaffrey CI (2006) Complications in spinal fusion for adolescent idiopathic scoliosis in the new millennium. A report of the Scoliosis Research Society Morbidity and Mortality Committee. Spine 31:345-9

4. Flinchum D (1963) Rib resection in the treatment of scoliosis. South Med J 56:1378-80

5. Goldstein LA (1969) American Academy of Orthopaedic Surgeons: Symposium on the spine. Concave rib resection and ligament release for correction of idiopathic scoliosis. St Louis, Mosby

6. Halsall AP, James DF, Kostuik JP, Fernie GR (1983) An experimental evaluation of spinal flexibility with respect to scoliosis surgery. Spine 8:482-8

7. Kostuik JP (1980) Recent advances in the treatment of painful adult scoliosis. Clin Orthop Relat Res 147:238-52

8. Mann DC, Nash CL Jr, Wilham MR, Brown RH (1989) Evaluation of the role of concave rib osteotomies in the correction of thoracic scoliosis. Spine 14:491-5

9. Masso PD, Gorton GE III (2000) Quantifying changes in standing body segment alignment following spinal instrumentation and fusion in idiopathic scoliosis using an optoelectronic measurement system. Spine 25:457-62

10. Schwend RM, Hennrikus W, Hall JE, Emans JB (1995) Childhood scoliosis: clinical indications for magnetic resonance imaging. J Bone Joint Surg Am 77:46-53

11. Stirling AJ, Howel D, Millner PA, Sadiq S, Sharples D, Dickson RA (1996) Late-onset idiopathic scoliosis in children six to fourteen years old. A cross-sectional prevalence study. J Bone Joint Surg Am 78:1330-6

12. Tolo VT (1989) Surgical treatment of adolescent idiopathic scoliosis. Instr Course Lect 38:143-56

13. Weiner P, Man A, Weiner M, Rabner M, Waizman J, Magadle R, Zamir D, Greiff Y (1997) The effect of incentive spirometry and inspiratory muscle training on pulmonary function after lung resection. J Thorac Cardiovasc Surg 113:552-7 